Deviations of the spin axes from the normal to the ecliptic are probably due to the statistical fluctuations in the final accumulation of any planet, in other words Uranus' unusual $98^{\circ}$ obliquity could be produced by the angular momenta brought in by the last few accreting planetismals. This statistical fluctuation in the accretion process also causes the scatter in the asteroid data.

Two other questions are of interest. First, do these collision-induced spin rates in asteroids ever become so rapid that the surface layers are not gravitationally bound? Excessive spin rates could sweep away any regolith and leave a clean surface. Burns concludes that this does not happen. Even in the case of 1566 Icarus, the smallest and fastest spinning asteroid of known rotation and shape, and 321 Florentina the second most rapid rotator, their densities have to be less than 2.8 and $2.3 \mathrm{~g} \mathrm{~cm}^{-3}$ respectively for this to occur. As asteroids seem to fall into two main compositional groups, however, (Chapman et al., Icarus, 25, 104; 1975) similar to carbonaceous chondrite meteorite (Type I has $\rho=2.5 \mathrm{~g} \mathrm{~cm}^{-3}$, Type II has $\rho=2.9 \mathrm{~g} \mathrm{~cm}^{-3}$ ) and to stony-iron meteorites $\left(\rho=5.0 \mathrm{~g} \mathrm{~cm}^{-3}\right)$ things are getting pretty close.

Second, are the spin rates fast enough to cause internal tensile stresses which over long periods of time, split the asteroids apart. Burns again concludes that the answer is no and that if forces are attractive on the surface of the asteroid they are also attractive throughout the interior.

In the past asteroids might have been spinning about other axes than that of maximum moment of inertia. The spin rate would then be faster and these excess rates could have peeled off the requisite outer layers to produce a stable configuration. The fact that carbonaceous chondrite material is very friable and that 1566 Icarus and 321 Florentina are surprisingly spherical for their sizes and rotation rates adds credence to this idea; however more observations of small asteroids are needed to confirm this thesis.

\section{Flipping rings in proteins}

\section{from G. C. K. Roberts.}

$X-R A Y$ crystallography has given us a very detailed picture of the structure of many proteins. This picture is, however, a static one, and in recent years there has been much interest in attempts to understand in detail the dynamics of protein structure. Nuclear magnetic resonance (NMR) spectroscopy is potentially able to give information on the rate of motion of individual amino-acid sidechains within a protein. The complexity of the NMR spectra of proteins often makes this information difficult to extract, but recently several reports have appeared which focus on the motional characteristics of the aromatic rings of tyrosine and phenylalanine residues.

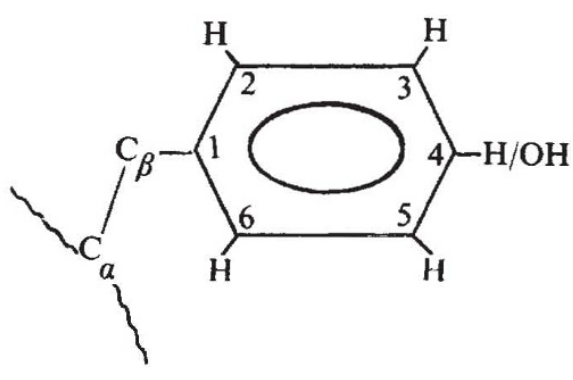

Sykes and his colleagues have been studying the alkaline phosphatase of Escherichia coli, labelled in vivo with 3-fluorotyrosine. The ${ }^{19} \mathrm{~F}$ NMR spectrum shows a separate resonance for each of the 11 tyrosine residues per subunit, and a thorough study of the ${ }^{19} \mathrm{~F}$ relaxation behaviour has allowed Hull and Sykes (J. molec. Biol., 98, 121, 1975) to draw a number of interesting conclusions about the motion of these tyrosine side chains. They observed that the linewidth of the ${ }^{19} \mathrm{~F}$ resonances (related to the relaxation time $T_{2}$ ) was markedly frequency dependent, becoming greater at higher frequency. One relaxation mechanism which would be expected to give rise to a frequency-dependent $T_{2}$ is chemical shift anisotropy relaxation. The chemical shift of a nucleus is a tensor quantity which depends on the orientation of the molecule with respect to the external magnetic field. In liquids, the tumbling of the molecule averages the chemical shift over all orientations, but the resulting fluctuations in Zeeman energy provide a relaxation mechanism which is most effective at high fields and for nuclei (such as ${ }^{19} \mathrm{~F}$ ) which have a large chemical shift anisotropy. The linewidths of 9 of the 11 fluorotyrosine resonances could be explained in this way, and this analysis led to estimates of the rate of internal motion about the $\mathrm{C}_{\mathrm{B}}-\mathrm{C}(1)$ bond ranging from $10^{6}$ to $10^{8} \mathrm{~s}^{-1}$ (the overall tumbling of the protein having a rate of about $2 \times 10^{6} \mathrm{~s}^{-1}$ ). For two of the tyrosines, there was an additional contribution to the linewidth from exchange, and for these the rate of reorientation of the ring was slower, in the range $10^{2}-5 \times 10^{4} \mathrm{~s}^{-1}$. Analysis of the spin-lattice relaxation time and of the dipolar contribution to $T_{2}$ led to an upper limit for motion about the $\mathrm{C}_{\alpha}-\mathrm{C}_{\beta}$ bond of $10^{6} \mathrm{~s}^{-1}$

In favourable circumstances, analysis of the ${ }^{1} \mathbf{H}$ spectrum of tyrosines and phenylalanines in proteins can give a rather direct indication of the rate of rotation about the $C_{\beta}-C(1)$ bond. This bond lies along the symmetry axis of the aromatic ring (see figure). In the free amino acids or simple peptides, the 2 and 6 protons and the 3 and 5 protons are equivalent, and a simple spectrum is observed. For a tyrosine or a phenylalanine in a protein, such a simple spectrum will be observed only if either the environment of the two sides of the ring is identical-an unlikely coincidence-or the ring is rotating sufficiently rapidly for the 2 and 6 and 3 and 5 protons, respectively, to experience an average environment. Roughly speaking, a rotation about $\mathrm{C}_{\beta}-\mathrm{C}(1)$ of approximately $200 \mathrm{~s}^{-1}$ will be sufficient to produce this averaging.

In hen lysozyme, all three tyrosine residues show simple spectra indicative of averaging about $\mathrm{C}_{\beta}-\mathrm{C}(1)$ (Campbell, Dobson and Williams, Proc. R. Soc. Lond., B189, 503-509; 1975). The bovine basic pancreatic trypsin inhibitor has four tyrosine residues, whose resonances have been assigned to individual residues in the sequence (Snyder et al., Biochemistry, 14, 3765; 1975). Three of these show simple spectra at room temperature, while the fourth, Tyr 35 , shows a complex spectrum indicative of slow rotation (Snyder et al., op. cit., Wüthrich, presented at Conference on Molecular Structural Methods in Biological Research, Stanford, Oct. 9-10, 1975). Similar phenomena are seen for the phenylalanine residues (Wüthrich and Wagner, FEBS Lett., 50, 265; 1975). Values for the activation energies can be obtained by temperature studies (Wüthrich, op. cit.).

It thus seems to be rather common for the aromatic rings of tyrosine and phenylalanine residues in proteins to rotate (the motion is in all probability essentially a flip through $180^{\circ}$ ) at rates greater than $200 \mathrm{~s}^{-1}$. Some of the implications of this are brought out in a recent paper by Gelin and Karplus (Proc. natn. Acad. Sci. U.S.A., 72, 2002-2006; 1975). These authors have calculated the energy barriers to rotation about $\mathrm{C}_{\beta}-\mathrm{C}(1)$ of the tyrosines of the basic trypsin inhibitor, based on its crystal structure, which is known to high resolution. For both the crystallographically determined structure and an energy-refined version thereof, the barriers are extremely

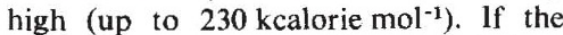
protein structure was allowed to 'relax', however, (simulated by energy minimisation with the tyrosine ring in its minimum and maximum energy orientations), a drastic reduction in the barriers could be achieved at the cost of a rather modest amount of bond angle strain. In this case, at least, it appears that a significant "breathing" of the protein is required to permit the aromatic rings to flip at the rates implied by the NMR experiments. 